\title{
Blends of Poly(mono $n$-alkyl itaconates) with Tertiary Polyamides: Specific Interactions and Thermal Degradation
}

\author{
Emilio Meaurio, Luis Cesteros, Luis G. Parada, and Issa Katime ${ }^{\dagger}$ \\ Grupo de Nuevos Materiales y Espectroscopia Supramolecular. Departamento de Química Física, Facultad de Ciencias, \\ Universidad del País Vasco, Campus de Leioa, Apartado 644. Bilbao, España
}

(Received June 16, 2003; Accepted November 19, 2003)

\begin{abstract}
The specific interactions in blends of poly(mono- $n$-alkyl itaconate)s with two tertiary polyamides, the poly( $N, N$-dimethylacrylamide) (PDMA) and the poly(ethyloxazoline) (PEOX), were studied. Hydrogen bonding between carboxylic acid groups of poly(mono- $n$-alkyl itaconate)s and carbonyl groups of PDMA and PEOX was found. The interassociation extent was measured by spectral fitting of the Amide I band and reveals a loss of interassociating ability in blends with PDMA as the length of the size group of the poly(mono- $n$-alkyl itaconate) increases, while in blends with PEOX the interassociation degree remains constant for a given composition. This different behavior is attributed to the larger interspacing between vicinal carbonyl groups in PEOX. Spectral changes upon heating showed an increase of thermal stability of the polyacid in the blends, larger in blends with PDMA than PEOX. These changes are explained in terms of the hydrogen bonding and the rigidity of the system. Finally, the ratio of absortion coefficients for the Amide I band was obtained.

KEY WORDS FTIR / Specific Interactions / Hydrogen Bonding / Poly(mono- $n$-alkyl itaconate)s / Poly( $N, N$-dimethylacrylamide) (PDMA) / Poly(ethyloxazoline) (PEOX) /
\end{abstract}

During the past years, the study of polymer blends as alternative materials has greatly expanded. Miscibility of polymer blends can only be achieved when the entalpy of mixing is negative, since the entropic contribution to the free energy of mixing is negligible. Therefore, specific interactions at the molecular level will promote the formation of miscible polymer blends.

Mixing of polyacids with polybases usually leads to hydrogen bonded miscible polymer blends. Poly(monomethyl itaconate) (PMMI) is an interesting polyacid due to its biotechnological origin that has been reported to be miscible with poly(ethyloxazoline) (PEOX) and poly $\left(N, N\right.$-dimethylacrylamide) (PDMA). ${ }^{1}$ In the present work, we are interested in expanding the miscibility study to poly(mono- $n$-alkyl itaconate)s with alkyl side groups ranging from methyl to dodecyl. These polymers contain the same donor group, but side groups of different length, and thus, different steric hindrances are expected. As specific interactions are the responsible for miscibility, their strength and extent are foundamental parameters to explain the miscibility behavior of these polymer blends. Moreover, their study has an additional interest: these polymer systems are known to form interpolymer complexes in the appropiate medium and temperature conditions, ${ }^{1,2}$ and further insight in their complexation behavior should be gained.

Thus, the present work deals with a FTIR study of the specific interactions present in the poly(mono- $n$ -

${ }^{\dagger}$ To whom correspondence should be addressed. alkyl itaconate)/PDMA or PEOX blends. The extent of the interassociation is determined by spectral curve fitting methods. In addition, the spectral behavior with respect to temperature has been studied, and the differences of thermal stability observed have been discussed.

\section{EXPERIMENTAL}

The polymerization of poly $(N, N$-dimethylacrylamide) (PDMA) has been reported in our previous paper. ${ }^{1}$ The molar mass of the polymer was measured viscometrically in a Ubbelohde type viscometer in methanol at $25^{\circ} \mathrm{C}$, using the relation: ${ }^{3}$

$$
[\eta]=1.75 \times 10^{-4} M_{\mathrm{v}}^{0.68}(\mathrm{dL} / \mathrm{g})
$$

which leads to $M_{\mathrm{v}}=2.9 \times 10^{5} \mathrm{~g} / \mathrm{mol}$.

Poly(ethyloxazoline) (PEOX) is a Polysciences product, and was purified by solution-precipitation in the methyethylketone/hexane pair. According to the supplier, the molar mass of the sample is $M_{\mathrm{w}}=5.0 \times 10^{4} \mathrm{~g} / \mathrm{mol}$.

Mono $n$-alkyl itaconates were obtained by the esterification of itaconic acid with the corresponding alcohol, using acetyl chloride as catalyst, according to a method previously described. ${ }^{4}$ The monomers were characterized by FTIR, ${ }^{1} \mathrm{H} N \mathrm{NR}$ and ${ }^{13} \mathrm{C}$ NMR. The poly(mono $n$-alkyl itaconates) used in this study, poly(monomethyl itaconate) PMMI, poly(monoethyl itaconate) PMEI, poly(monobutyl itaconate) PMBuI, poly(monohexyl itaconate) PMHeI, poly(monodecyl itaconate) PMDeI, and poly(monododecyl itaconate) 
Table I. Molar masses of the Poly(mono $n$-alkyl itaconate)s

\begin{tabular}{cc}
\hline Polymer & $M_{\mathrm{w}}(\mathrm{g} / \mathrm{mol})$ \\
\hline PMMI & $1.3 \times 10^{5}$ \\
PMEI & $2.5 \times 10^{5}$ \\
PMBuI & $3.5 \times 10^{5}$ \\
PMHeI & $3.9 \times 10^{5}$ \\
PMDeI & $1.1 \times 10^{5}$ \\
PMDoI & $9.2 \times 10^{4}$ \\
\hline
\end{tabular}

PMDoI, were obtained by free radical bulk polymerization using $0.3 \%$ AIBN as initiator under $\mathrm{N}_{2}$ atmosphere at 60 or $80^{\circ} \mathrm{C}$, depending on the melting temperature of the monomer. The polymers were characterized by light scattering in methanol or tetrahydrofurane solutions, at $25.0^{\circ} \mathrm{C}$. The obtained molar masses are summarized in Table I.

The blends were obtained by solvent casting from solutions with a total concentration of $2 \% \mathrm{wt} / \mathrm{v}$. Chosen solvents were DMF (system PMMI/PDMA), ethylene glycol (system PMMI/PEOX), methanol (blends with PMEI, PMBuI and PMHeI), butanol (blends with PMDeI) and pentanol (blends with PMDoI). In all cases the solvent was cast at $50^{\circ} \mathrm{C}$.

Infrared spectra were recorded on a Nicolet-520 Fourier transform infrared spectrophotometer (FTIR). Spectra were taken with a resolution of $2 \mathrm{~cm}^{-1}$ and were averaged over 100 scans. Blend samples were prepared on $\mathrm{KBr}$ pellets, using the following procedure. First, the polymer solution was cast in a petri dish at $50{ }^{\circ} \mathrm{C}$ under vacuum if necessary. When the system reached a gel-like state, it was spreaded between two KBr pellets in order to obtain a film of homogeneous thickness. The upper pellet was then removed and the sample was vacuum dried at $65^{\circ} \mathrm{C}$ for $72 \mathrm{~h}$. The absorbance of all the studied samples obeyes the Lambert-Beer law. High-temperature experiments were performed using a Specac variabletemperature cell $\mathrm{P} / \mathrm{N} 21500$ with a Eurotherm 847 temperature unit controller coupled to the spectrophotometer.

DSC analysis was performed in a Mettler TA4000 system. The weight of the samples was about $8 \mathrm{mg}$ in all cases and the scan speed was $10^{\circ} \mathrm{C} / \mathrm{min}$ under a nitrogen atmosphere $(200 \mathrm{~mL} / \mathrm{min})$.

\section{EXPERIMENTAL RESULTS}

\section{Analysis of Infrarred Spectra}

Poly(mono- $n$-alkyl itaconate)s with alkyl groups ranging from methyl to dodecyl (represented by a $\mathrm{R}$ in the chemical formula) include a carboxylic acid and a ester group in each repetitive unit: we are dealing with self-associated polymers.

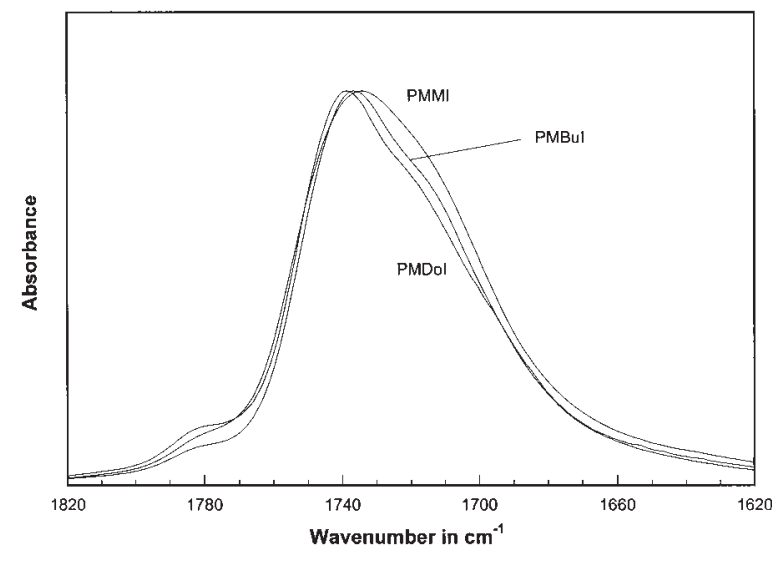

Figure 1. Autoscaled carbonyl stretching bands for PMMI, PMBuI and PMDoI.<smiles>CCC(CC)(CC(=O)O)C(=O)O</smiles>

Figure 1 shows the carbonyl stretching region for PMMI, PMBuI and PMDoI, which presents a rather complex profile due to the overlapping of several bands. It is interesting to describe its major features in order to understand the modifications observed upon blending. Reported spectral data for methacrylic acid, ${ }^{5}$ indicate that ester group contribution occurs at about $1735 \mathrm{~cm}^{-1}$. The carboxylic acid group can exist nonassociated (band at about $1750 \mathrm{~cm}^{-1}$ ), but most of it tends to be associated, in the form of carboxylic acid dimers (band at about $1700 \mathrm{~cm}^{-1}$ ). ${ }^{5}$ The spectra show that as the size of the side group of the poly(mono- $n$ alkyl itaconate) increases, the relative absorption due to carboxylic acid dimers decreases. Finally, the small contributions at about 1780 and $1850 \mathrm{~cm}^{-1}$ are attributed to residual anhydrides, formed during the polymerization process. ${ }^{6,7}$ PDMA and PEOX are polymers containing tertiary amide groups in the chemical structure of their repetitive unit.
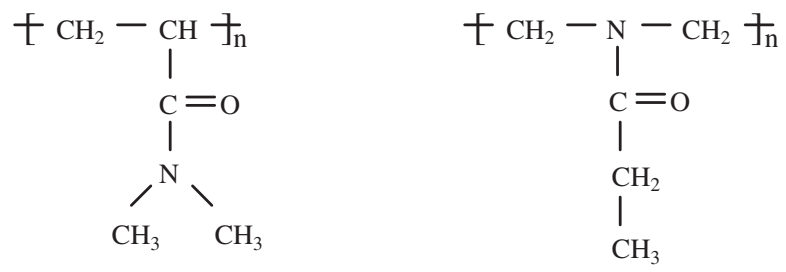

Studies performed with model compounds show that hydrogen bonding in these. polymers takes mainly place with the carbonyl group. ${ }^{89}$ The absortion band in the carbonyl stretching region for these polymers is called Amide I and is a composed mode of the $\mathrm{C}=\mathrm{O}$ and $\mathrm{C}-\mathrm{N}$ stretching modes, with a larger contri- 


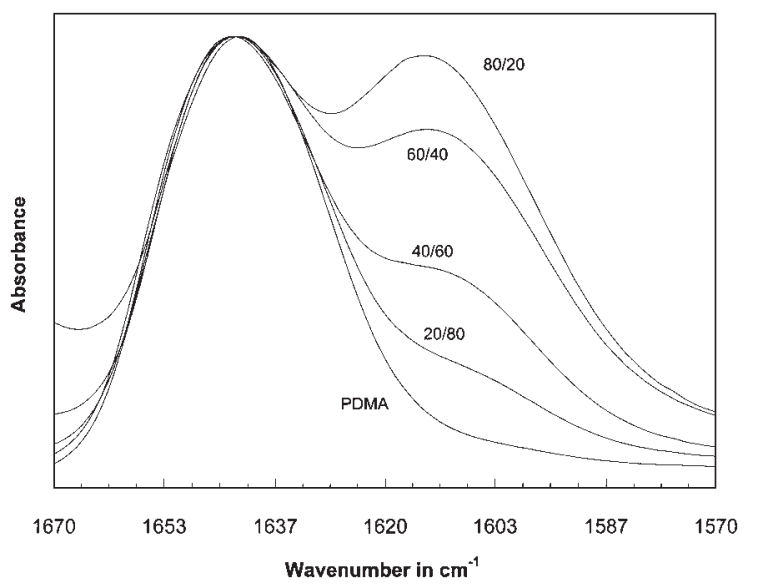

Figure 2. Autoscaled carbonyl stretching bands for PDMA and PMBuI/PDMA blends with different wt/wt composition.

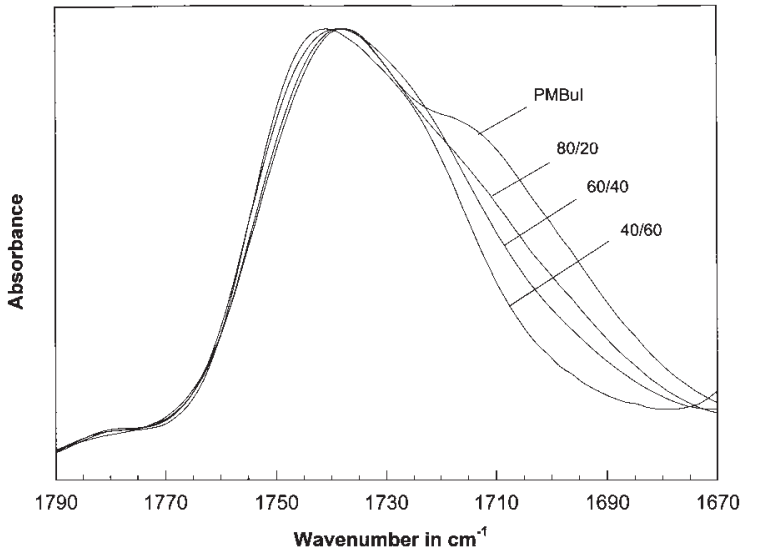

Figure 3. Autoscaled carbonyl stretching bands for PMBuI and PMBuI/PDMA blends with different wt/wt composition.

bution of the first one. ${ }^{10}$

Figure 2 shows the carbonyl stretching region for PMBuI/PDMA blends, obtained from methanol solutions. Spectral inspection shows the presence of a new band at about $1610 \mathrm{~cm}^{-1}$, that can be assigned to PDMA hydrogen-bonded carbonyl groups. Figure 3 shows the PMBuI carbonyl band region for the same system. In this case, increasing the content of PDMA produces a steadily loss of the relative intensity of the contribution located at about $1700 \mathrm{~cm}^{-1}$. These results suggest that blending breaks the carboxylic acid dimers, and permits hydrogen bond formation between the carboxylic acid and the amide groups. This behavior matches qualitatively the obtained by our research group in PMMI/PDMA blends ${ }^{1}$ and by Lichkus et al. in poly(ethylene-co-methacrylic acid)/PEOX blends. ${ }^{5}$

Similar results were obtained for blends with PEOX. As can be seen in Figure 4, addition of PMDeI to PEOX results again in a new band at around $1610 \mathrm{~cm}^{-1}$, with an intensity dependent on composition. The behavior of the $1700 \mathrm{~cm}^{-1}$ band is similar to that of PMBuI/PDMA blends. Thus, the infrared results suggest that the mechanisms responsible for

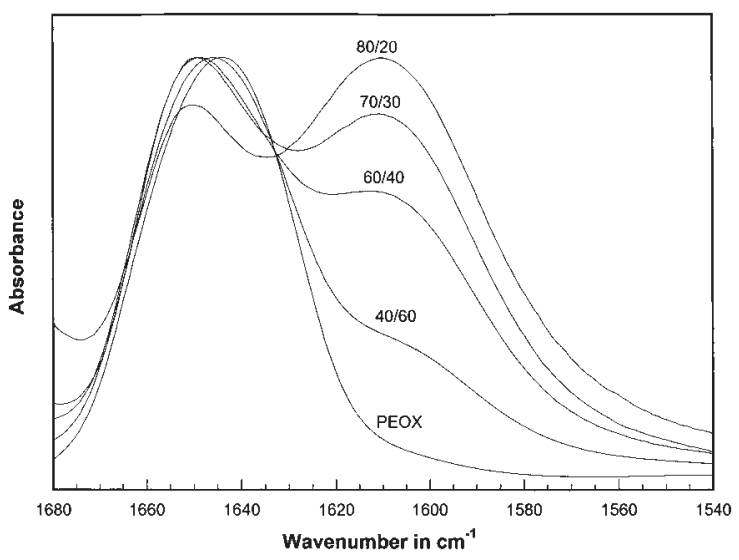

Figure 4. Autoscaled carbonyl stretching bands for PEOX and PMDeI/PEOX blends with different wt/wt composition.

miscibility are very similar in blends with PDMA or PEOX. Furthermore, the spectral shift observed in both cases is similar. Considering the relation between spectral shift and bond strength, ${ }^{11}$ it suggests a similar bond strength in both cases.

\section{Spectral Curve Fitting}

The percentage of PDMA and PEOX carbonyl groups associated and nonassociated can be determined by using spectral curve fitting methods. The reliability of the results depends mainly on the suitability of the mathematical model chosen for the fitting and on the resolution of the overlapping bands. ${ }^{12}$ Gaussian line shape function gives a good fitting for the pure components as well as for the blends, and fortunately, the carbonyl stretching region of PEOX and PDMA in the blends studied is fairly well resolved. Tables II and III show the spectral fitting results obtained for the carbonyl stretching bands of PDMA and PEOX on blends with a composition polyacid/ polybase $60 / 40 \mathrm{~mol} / \mathrm{mol}$.

From the calculated values of the absorbance of the associated and nonassociated contributions it is possible to determine the fraction of nonassociated carbonyl groups $f_{\mathrm{CO}}^{\mathrm{F}}$ :

$$
f_{\mathrm{CO}}^{\mathrm{F}}=\frac{A_{\mathrm{F}}}{A_{\mathrm{F}}+\frac{\varepsilon_{\mathrm{F}}}{\varepsilon_{\mathrm{A}}} A_{\mathrm{A}}}
$$

The procedure requires the ratio of molar absorption coefficients of both spectral contributions. The temperature behavior of the studied systems leads to a value $\varepsilon_{\mathrm{F}} / \varepsilon_{\mathrm{A}}=1.0$, as discussed later on.

The values obtained for $f_{\mathrm{CO}}^{\mathrm{F}}$ in the different systems are ploted in Figure 5. As can be seen, blends with PDMA show an interassociating ability loss as the size of the side group of the poly(mono- $n$-alkyl itaconate) increases (see Figure 5). The different behavior shown by PDMA and PEOX can not be attributed to 
Polymer Blends

Table II. Curve-fitting results of the PDMA carbonyl stretching band in a blend $60 / 40 \mathrm{~mol} / \mathrm{mol}$

\begin{tabular}{ccccccccc}
\hline & \multicolumn{3}{c}{ Free $\mathrm{C}=\mathrm{O}$ band } & & \multicolumn{3}{c}{ Bonded $\mathrm{C}=\mathrm{O}$ band } \\
\cline { 2 - 3 } Polyacid & $\begin{array}{c}\text { Wavenumber } \\
\left(\mathrm{cm}^{-1}\right)\end{array}$ & $\begin{array}{c}\text { Width } \\
\left(\mathrm{cm}^{-1}\right)\end{array}$ & rel area & & $\begin{array}{c}\text { Wavenumber } \\
\left(\mathrm{cm}^{-1}\right)\end{array}$ & $\begin{array}{c}\text { Width } \\
\left(\mathrm{cm}^{-1}\right)\end{array}$ & rel area & $f_{\mathrm{F}}$ \\
\hline - & 1642 & 30 & 100 & & & & & 1 \\
PMMI & 1643 & 26 & 30 & & 1610 & 40 & 70 & 0.30 \\
PMEI & 1643 & 26 & 33 & & 1611 & 44 & 67 & 0.33 \\
PMBuI & 1644 & 26 & 35 & & 1612 & 44 & 65 & 0.35 \\
PMHeI & 1644 & 26 & 38 & & 1612 & 44 & 62 & 0.38 \\
PMDeI & 1645 & 26 & 43 & & 1611 & 38 & 57 & 0.43 \\
PMDoI & 1645 & 26 & 43 & & 1611 & 38 & 57 & 0.43 \\
\hline
\end{tabular}

Table III. Curve-fitting results of the PEOX carbonyl stretching band in a blend $60 / 40 \mathrm{~mol} / \mathrm{mol}$

\begin{tabular}{ccccccccc}
\hline & \multicolumn{3}{c}{ Free $\mathrm{C}=\mathrm{O}$ band } & & \multicolumn{3}{c}{ Bonded $\mathrm{C}=\mathrm{O}$ band } \\
\cline { 2 - 3 } Polyacid & $\begin{array}{c}\text { Wavenumber } \\
\left(\mathrm{cm}^{-1}\right)\end{array}$ & $\begin{array}{c}\text { Width } \\
\left(\mathrm{cm}^{-1}\right)\end{array}$ & rel area & & $\begin{array}{c}\text { Wavenumber } \\
\left(\mathrm{cm}^{-1}\right)\end{array}$ & $\begin{array}{c}\text { Width } \\
\left(\mathrm{cm}^{-1}\right)\end{array}$ & rel area & $f_{\mathrm{F}}$ \\
\hline- & 1643 & 37 & 100 & & & & & 1 \\
PMMI & 1650 & 37 & 27 & & 1608 & 52 & 76 & 0.27 \\
PMEI & 1650 & 37 & 33 & & 1607 & 52 & 67 & 0.33 \\
PMBuI & 1650 & 36 & 35 & & 1608 & 50 & 65 & 0.35 \\
PMHeI & 1650 & 35 & 33 & & 1608 & 50 & 66 & 0.33 \\
PMDeI & 1653 & 30 & 31 & & 1609 & 50 & 69 & 0.31 \\
PMDoI & 1654 & 30 & 31 & & 1610 & 50 & 69 & 0.31 \\
\hline
\end{tabular}

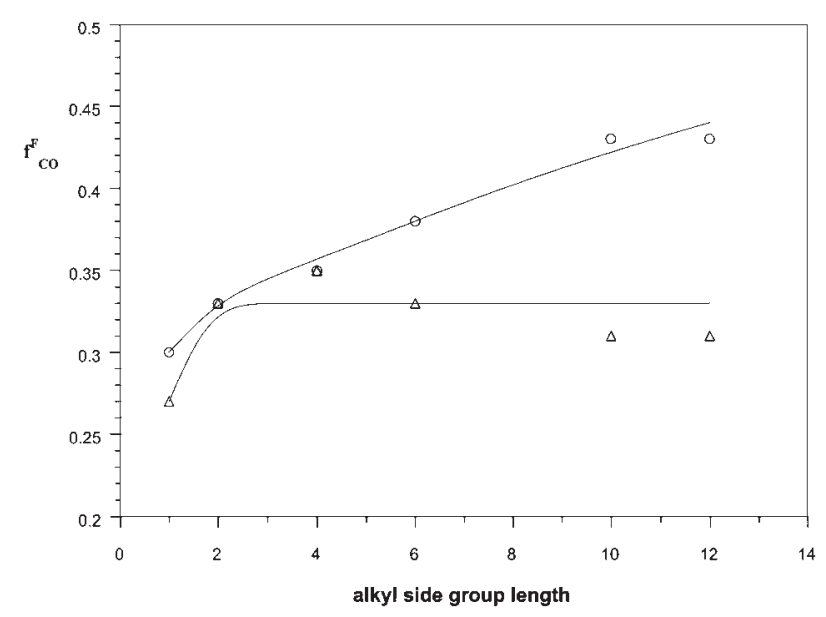

Figure 5. Fraction of free carbonyl groups versus the length of the side group of the poly(mono- $n$-alkyl itaconate) in blends of composition polyacid/polybase $60 / 40 \mathrm{~mol} / \mathrm{mol}$ : (O) systems with PDMA and $(\triangle)$ systems with PEOX.

different steric hindrance around the acceptor groups: these are located at the same distance from the polymeric chain, and the chemical groups surrounding them are not likely to impair hydrogen bonding. Moreover, according to the measured spectral shifts, both carbonyl groups have similar acceptor strengths and in fact show similar interassociation extents when blending with poly(mono- $n$-alkyl itaconate)s of small side groups. Such difference may be due to the different interspacing between neighbouring interacting sites. In PDMA, there are four covalent bonds between two vicinal carbonyl groups, while in PEOX there are five. This circumstance gives more degrees of freedom to the PEOX chain, and so a higher ability to adapt its conformation and establish as many hydrogen bonds as possible when the steric hindrances present in the medium increase.

In connection with these results, it was found that as the side group size of the poly(monoalkyl itaconate) increases, the relative stability in solution of the interpolymer complexes with PEOX compared to that of the complexes with PDMA increases. ${ }^{2}$ Now the FTIR results explain that behavior.

A final remark concerns to the width of the PEOX free carbonyl band. When a polymer is mixed, the form of any infrared band can change due to environmental or conformational changes related to blending. ${ }^{13}$ The amide group in PEOX is directly linked to the polymeric chain, and it is expected some conformational sensitivity for the Amide I absorption band. As can be seen in Table III, blending with poly(mono- $n$-alkyl itaconate)s of larger size groups leads to narrower PEOX free carbonyl bands than those obtained when the polyacid has not bulky side groups. Narrower bands are typical of systems with higher structural regularity. It seems that blending with poly(mono $n$-alkyl itaconates) of bulky side group forces the PEOX chain to adopt a more regular and probably constrained conformation.

\section{Temperature Behavior}

The thermal behavior of the blends has been studied in the range of $50-250{ }^{\circ} \mathrm{C}$ in samples of stoichiometric 


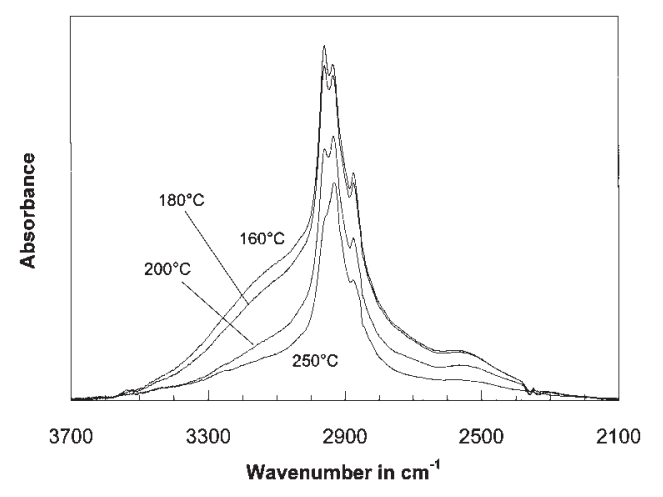

Figure 6. Hydroxyl stretching region spectra of the PMBuI/ PDMA 60/40 wt/wt blend at different temperatures.

composition, being the heating rate $5^{\circ} \mathrm{C} / \mathrm{min}$ in all cases. For all the samples studied, strong alterations are observed in different spectral regions related to hydrogen-bond breaking and to poly(mono- $n$-alkyl itaconate)s thermal degradation. The hydroxyl stretching region, represented in Figure 6, undergoes an evolution with temperature characterized by a progressive decrease in absorbance. This effect can be explained by the progressive loss of hydroxyl groups (in the form of the corresponding alcohol or water) due to anhidride formation.

Anhydride formation generates well known changes in the carbonyl stretching region. ${ }^{14-17}$ Thus, as can be seen from Figure 7, an increase of the intensity of the bands corresponding to ciclyc anhydrides (five member rings at around 1850 and $1780 \mathrm{~cm}^{-1}$, and six member rings at around 1800 and $1760 \mathrm{~cm}^{-1}$ ) is observed. Simultaneously a gradual reduction in the absorbance of other types of carbonyl stretching vibrations occurs. In any system, the intensities of the carbonyl bands corresponding to cyclic anhydrides begin to decrease at certain temperature, this behavior could be explained in terms of decarboxilation through $\mathrm{CO}_{2}$ formation. Moreover, physical

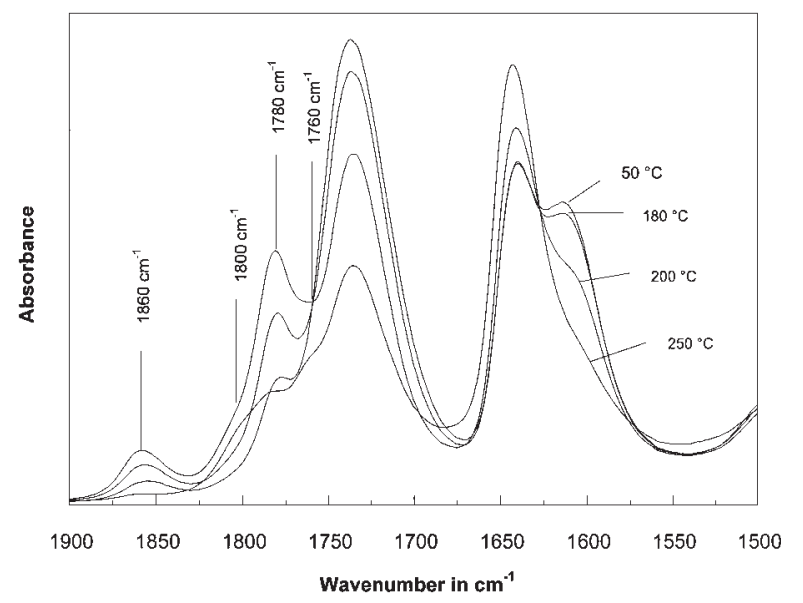

Figure 7. Carbonyl stretching region spectra of the PMMI/ PDMA 60/40 wt/wt blend at different temperatures.

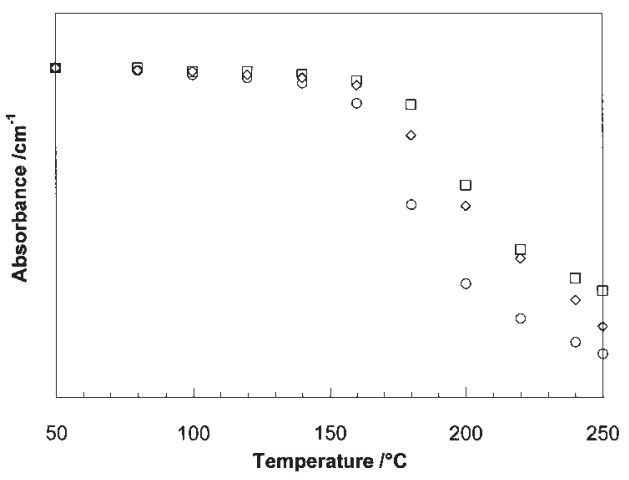

Figure 8. Absorbance at $1735 \mathrm{~cm}^{-1}$ vs. temperature: (O) PMBuI, $(\diamond)$ PMBuI/PEOX 60/40 wt/wt blend and $(\square)$ PMBuI/PDMA 60/40 wt/wt blend.

crosslinking of the samples occurs, as probed from solubility tests, suggesting the formation of linear anhydrides. ${ }^{14,16}$ Both processes (decarboxylation and anhydride formation) yield a decrease of the carbonyl vibration band intensity. This change can be used as a measure of the rate of degradation of the poly(mono- $n$-alkyl itaconate). Figure 8 shows the absorbance at $1735 \mathrm{~cm}^{-1}$ (where carbonyl stretching peak maximum occurs) versus temperature for pure $\mathrm{PMBuI}$ and its blends of polyacid/polybase 60/40 wt/wt composition. As can be seen, blending with PDMA or PEOX increases the thermal stability of the poly(mono- $n$-alkyl itaconate). The onset of the degradation process are of about $157^{\circ} \mathrm{C}$ for PMBuI, $165^{\circ} \mathrm{C}$ for the PMBuI/PEOX blend and $173^{\circ} \mathrm{C}$ for the PMBuI/PDMA blend. The other systems show a similar general trend: the onset in blends with PEOX is 5$10^{\circ} \mathrm{C}$ higher than the one of the pure poly(mono- $n$-alkyl itaconate) and $15-20^{\circ} \mathrm{C}$ in blends with PDMA.

The reported data suggest that the thermal degradation behavior is mainly controlled by the hydrogen bonding in the system. Anhydride formation is the first degradation process, and involves the hydroxyl placed into the carboxylic acid groups of the poly(mono- $n$-alkyl itaconate). In the blends, a large number of hydroxyl groups are strongly hydrogen bonded to PDMA or PEOX. For these groups, the first step before the anhydride formation must be the break of hydrogen bonds. Thus, as the strength and extension of hydrogen bonds is more important in a particular sample, its break is more difficult and requires more thermal energy. Another important factor to take into account is the particular glass transition of the system. A lower glass transition in the system allows greater motion in the system, favoring the contacts for anhydride formation and also the breaking of specific interactions. Unfortunately, DSC scans in pure poly(mono$n$-alkyl itaconate)s and in the blends do not show any Tg. ${ }^{1}$ Nevertheless, the Tg measured for PDMA and PEOX are placed at 130 and $61{ }^{\circ} \mathrm{C}$ respectively. 
According to the general behavior in hydrogen-bonded blends, higher rigidity can be expected for systems with PDMA than for those with PEOX, and in fact the first systems exhibit higher degradation onset temperatures.

The spectral changes in the carbonyl band of PDMA and PEOX can be used to obtain the molar absorption coefficients ratio (Eq 2). This is only possible if the following conditions are fulfilled: i) the spectral change must be important, in order to diminish errors and ii) the sample amount absorbing in the region must not change. ${ }^{18}$ The first condition is clearly filled in our systems, and the second one too: pure PDMA and PEOX are known to be stable at temperatures up to $350^{\circ} \mathrm{C}$, and thermogravimetric analysis shows ${ }^{1}$ that its degradation process in blends with PMMI does not proceed below $300^{\circ} \mathrm{C}$. Figure 9 shows the spectra of the PMMI/PEOX $60 / 40$ blend at $50{ }^{\circ} \mathrm{C}$ before and after the thermal treatmet at $250^{\circ} \mathrm{C}$. Similar changes to those obtained in the PMMI/PDMA system are observed. Spectral fitting leads to the results summarized in Table IV, leading to a value $\varepsilon_{\mathrm{F}} / \varepsilon_{\mathrm{A}}=1.0$ in both type of systems. This result implies that the total area in the studied region remains unchanged, as can be verified by integration.

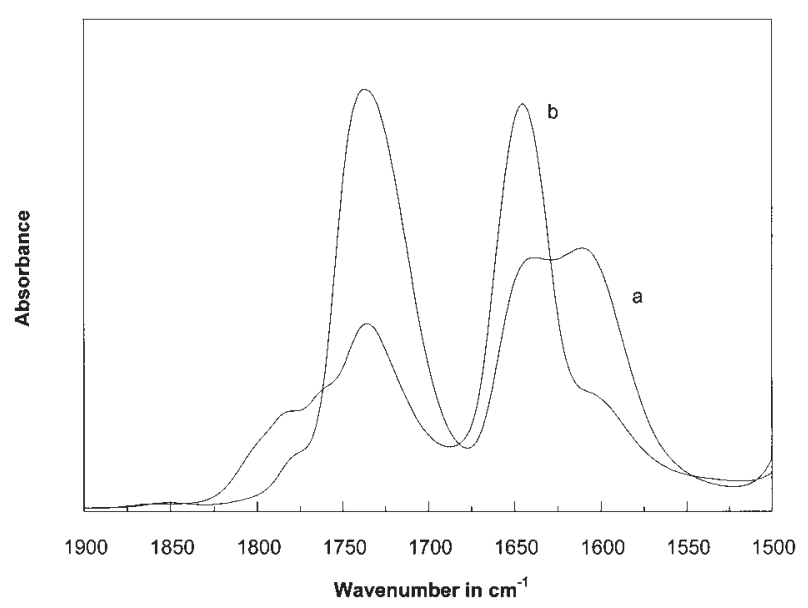

Figure 9. Carbonyl region spectra of the PMMI/PEOX 60/40 $\mathrm{wt} / \mathrm{wt}$ blend at $50^{\circ} \mathrm{C}$. a) before and b) after the thermal treatment at $250^{\circ} \mathrm{C}$.

$$
\frac{\varepsilon_{\mathrm{A}}}{\varepsilon_{\mathrm{L}}}=\frac{A_{\mathrm{A}, 1}-A_{\mathrm{A}, 2}}{A_{\mathrm{L}, 2}-A_{\mathrm{L}, 1}}
$$

\section{CONCLUSIONS}

Specific interactions when poly(monoalkyl itaconate)s are blended with poly(ethyloxazoline) and poly $(N, N$-dimethylacrylamide), and their evolution with temperature have been studied. Hydrogen bonding occurs between carboxylic acid groups of poly(monoalkyl itaconate)s and carbonyl groups of PDMA and PEOX. Infrarred results suggest too similar strengths in hydrogen bonds with PDMA or with PEOX.

The interassociation extent has been measured by spectral fitting of the Amide I band and reveals a loss of interassociating ability in blends with PDMA as the length of the side group of the poly(mono- $n$-alkyl itaconate) increases, while in blends with PEOX the interassociation degree remains constant for a given composition. This different behavior is attributed to the larger interspacing between vicinal carbonyl groups in PEOX, resulting in higher chain conformational freedom degrees. Anyway, the PEOX chain seems to be forced to adopt more regular and constrained conformations as the steric hindrances in the medium increase, as reflected by the variation of its free carbonyl band width.

The interassociation extent does not change upon heating below the temperature at which poly(mono$n$-alkyl itaconate)s degradation is observed. It can be attributed to the high rigidity of these systems. Degradation of the poly(mono- $n$-alkyl itaconate)s proceeds through anhydride formation and gives place to an important interassociation loss. These changes have been used to obtain the ratio of molar absorption coefficients of the Amide I band.

Finally, it has been observed that blending increases the thermal stability of the poly(mono- $n$-alkyl itaconate)s. The retardation of the degradation process is higher in blends with PDMA than in blends with PEOX. The thermal stability of these systems seems

Table IV. Curve-fitting results of carbonyl Stretching bands in blends $60 / 40 \mathrm{wt} / \mathrm{wt}$ at $50{ }^{\circ} \mathrm{C}$ obtained a) before and b) after the thermal treatment at $250^{\circ} \mathrm{C}$

\begin{tabular}{cccccccc}
\hline \multirow{2}{*}{ Blend } & \multicolumn{3}{c}{ Free $\mathrm{C}=\mathrm{O}$ band } & & \multicolumn{3}{c}{ Bonded $\mathrm{C}=\mathrm{O}$ band } \\
\cline { 2 - 3 } & $\begin{array}{c}\text { Wavenumber } \\
\left(\mathrm{cm}^{-1}\right)\end{array}$ & $\begin{array}{c}\text { Width } \\
\left(\mathrm{cm}^{-1}\right)\end{array}$ & rel area & & $\begin{array}{c}\text { Wavenumber } \\
\left(\mathrm{cm}^{-1}\right)\end{array}$ & $\begin{array}{c}\text { Width } \\
\left(\mathrm{cm}^{-1}\right)\end{array}$ & rel area \\
\hline PMMI/PDMA (a) & 1643 & 27 & 32.8 & & 1612 & 44 & 67.2 \\
PMMI/PDMA (b) & 1643 & 29 & 63.6 & & 1610 & 44 & 36.4 \\
PMMI/PEOX (a) & 1647 & 37 & 35.0 & & 1607 & 52 & 65.0 \\
PMMI/PEOX (b) & 1646 & 35 & 71.2 & & 1601 & 52 & 28.8 \\
\hline
\end{tabular}


to be controlled by hydrogen bonding and by the rigidity of the system.

Acknowledgment. The authors thanks the MCYT (MAT2000-0768-CO2-02) and the Universidad del País Vasco (UPV/EHU 00039.310-13635/2001) for their financial support.

\section{REFERENCES}

1. E. Meaurio, J. L. Velada, L. C. Cesteros, and I. Katime, Macromolecules, 29, 4598 (1996).

2. E. Meaurio, L. C. Cesteros, and I. Katime, Macromolecules, 30, 4567 (1997).

3. L. Trossarelli and M. Meirone, J. Polym. Sci., 57, 445 (1962).

4. L. C. Cesteros, E. Meaurio, and I. Katime, Polym. Int., 34, 97 (1994).

5. A. M. Lichkus, P. C. Painter, and M. M. Coleman, Macromolecules, 21, 2636 (1988).

6. S. Nagai and F. Fujiwara, J. Polym. Sci., Polym. Lett., 7, 177 (1969).
7. J. L. Velada, C. Cesteros, A. Madoz, and I. Katime, Macromol. Chem. Phys., 196, 3171 (1995).

8. C. D. Schmulbach and R. S. Drago, J. Phys. Chem., 64, 1956 (1960).

9. W. E. Bull, S. K. Madan, and J. E. Willis, Inorg. Chem., 2, 303 (1963).

10. V. Venkata Chalapathi and K. Venkata Ramiah, Curr. Sci., 16, 453 (1968).

11. T. K. Kwei, E. M. Pearce, F. Ren, and J. P. Chen, J. Polym. Sci., Part B: Polym. Phys., 24, 1597 (1986).

12. W. F. Maddams, Appl. Spectrosc., 34, 245 (1980).

13. P. Musto, L. Wu, F. E. Karasz, and W. J. Macknight, Polymer, 32, 3 (1991).

14. J. L. Velada, L. Cesteros, E. Meaurio, and I. Katime, Polymer, 36, 2765 (1995).

15. J. M. G. Cowie, Pure Appl. Chem., 51, 2331 (1979).

16. J. L. Velada, L. Cesteros, A. Madoz, and I. Katime, Macromol. Chem. Phys., 196, 3171 (1995).

17. J. L. Velada, E. Hernáez, C. Cesteros, and I. Katime, Polym. Deg. Stab., 52, 273 (1996).

18. J. Y. Lee, P. C. Painter, and M. M. Coleman, Macromolecules, 21, 346 (1988). 